Mediterránea Ser. Biol. (1987), n. ․ 9. Pág. 15-27

\title{
DATOS SOBRE ACTIVIDAD DE ANFIBIOS Y PEQUEÑOS REPTILES DE DOÑANA (HUELVA, ESPAÑA)
}

\author{
por \\ Carmen Díaz Paniagua ${ }^{1}$ y Rosario Rivas ${ }^{1}$
}

\section{RESUMEN}

El estudio se ha realizado en base a la observación y captura de individuos mediante dos métodos: 1) censos nocturnos usado sólo para los anfibios, 2) control de una serie de losetas, dispuestas previamente, bajo las que se cobijan anfibios y reptiles.

Para los anfibios, la mayor actividad se concentra en los meses más lluviosos, mientras que parece existir un período de inactividad durante el verano. Los dos métodos empleados detectan sólo la actividad terrestre, por lo que las especies de actividad muy ligada al medio acuático aparecen escasamente representadas.

Entre les reptiles, la mayoría de las observaciones corresponden a Podarcis hispanica, que llega a instalarse bajo las losas, así como Blanus cinereus que aparece en menor número. Otras especies, por el contrario, las utilizan solamente como un refugio transitorio. Se describen algunas características del hábitat utilizado por las especies observadas.

\section{SUMMARY}

The study was carried out throughout two different methods: 1) Night census of individuals, from a car at a constant slow speed, used only for amphibians, 2) Cobtrol of individuals refuged under flag-stones placed in the study area.

Main activity of amphibians occurs in most rainy months, while a no activity period seems to occur in summer months. The methods employed detected terrestrial activity of animals, thus a poor information was obtained on species of aquatic activity.

Most information recorded on reptiles is about Podarcis hispanica, a species which can use the tiles as a long time refugre. Blanus cinereus is considered as an usual dweller under the tiles, despite the number of individual captured is not large enough. Other species only inhabit them as a temporary refuge, appearing sporadically. Some habitat characteristics of species recorded are described.

(1) Estación Biológica de Doñana. P. M. ${ }^{a}$ Luisa. Pabellón del Perú. Saille. 


\section{INTRODUCCIÓN}

Es evidente que la actividad de anfibios y reptiles como animales ectotérmicos, está muy relacionada con las condiciones del medio que habitan. Las fluctuaciones climáticas estacionales y diarias condicionan, por tanto, fuertemente su ciclo de vida, presentańdose largos períodos de inactividad cuando las condiciones son adversas. En la mayoría de las especies es habitual el uso de refugios en los que se cobijan durante tales períodos, mientras que por el contrario, la actividad se limita a los momentos más favorables.

En el caso de los anfibios, la actividad nocturna de las especies más terrestres se considera una adaptación de su comportamiento encaminada a evitar la deshidratación, mientras que algunas especies mantienen una actividad diurna mediante su proximidad al medio acuático (ver, por ejemplo, DUELLMAN y TRUEB, 1985). En el caso de los reptiles, es de gran importancia la adquisición de una determinada temperatura corporal, la cual regulan, además de fisiológicamente, mediante un comportamiento dirigido a la búsqueda o alejamiento de las fuentes de calor (ver, por ejemplo, SPELLERBERG, 1982).

Las distintas adaptaciones del comportamiento en función de las características ambientales dificultan el empleo de métodos dirigidos a la observación o captura de anfibios y reptiles (VOGT y HINE, 1982). De tal manera, la diferente aceptación de un método, por parte de las especies consideradas, puede conducir a unos resultados que no reflejan necesariamente las relaciones de densidad interespecíficas. En este trabajo se ha pretendido obtener información sobre la actividad de anfibios y reptiles del área de Doñana. Los propios métodos elegidos determinan una selección de las especies, tanto por su actividad terrestre, en el caso de las anfibios, como por el tamaño en ambas clases consideradas. Por ello, no están incluidas todas las especies de anfibios y reptiles presentes en el área, quedando excluidas incluso algunas de gran abundancia.

\section{MATERIAL Y METODOS}

El estudio se ha llevado a cabo desde septiembre de 1982 a septiembre de 1984 (ambos incluidos) en la Reserva Biológica de Doñana (Huelva, España), zona que ha sido descrita ampliamente por varios autores (VALVERDE, 1967; ALLIER y col, 1974; GARCÍA NOVO y col, 1978; AMAT y col, 1979). En líneas generales, arenas estabilizadas y dunas móviles. Las marismas se caracterizan por su sustrato arcilloso, su escaso relieve y por la inundación que suele sufrir temporalmente, quedando entonces en ella pequeñas zonas que por su elevación podrán funcionar a modo de islas en la época de la inundación, mientras que otras zonas más deprimidas, se mantienen inundadas durante todo el año. En las arenas se distinguen unas áreas elevadas llamadas naves, constituidas por dunas estabilizadas, en las que la vegetación se caracteriza por la abundancia de Cistus libanotis, Juniperus phoenicea, Halimium commutatum, Lavandula stoechas, Thymus mastichina, etc. En esta zona no suelen formarse charcas temporales durante otoño-invierno. En el resto de las arenas estabilizadas el nivel de la capa freática se encuentra más próximo pudiéndose formar innumerables charcas temporales. En cuanto a la vegetación, predomina 


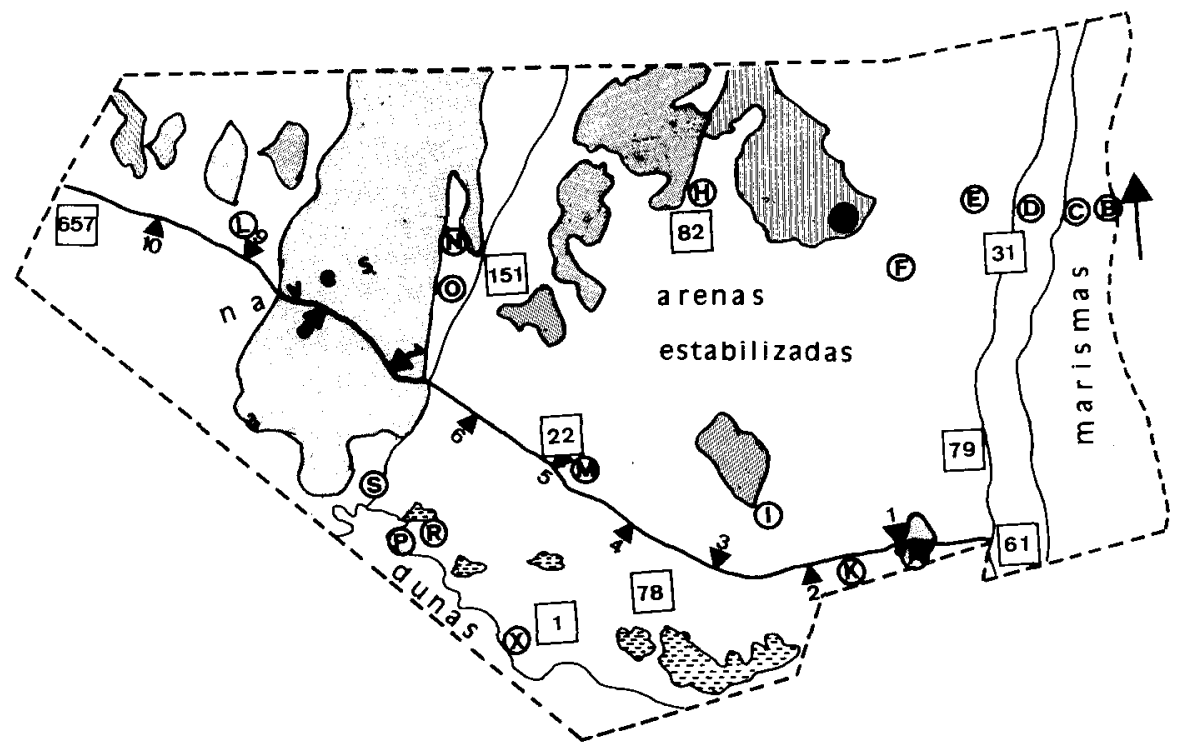

Figura 1.-Mapa de la Reserva Biológica de Doñana donde se detalla el recorrido de los censos (trazo grueso), y la localización de cada una de las series de losas (círculos). Se detalla también la distancia de la capa freática (en $\mathrm{mm}$.) en diciembre de 1984. La zona sombreada corresponde a pinares de $P$. Pinea, la zona rayada a lagunas.

Halimium halimifolium, y en las partes más húmedas Érica australis y Calluna vulgaris. Por último, en el sistema de dunas móviles, existen unas zonas interdunares en las que la capa freática está muy próxima, formándose también numerosas charcas y lagunas en las que predominan Holoschoenus vulgaris y diversas especies de Juncus, entre grupos de Pinus pinea.

Mensualmente, se registró la profundidad de la capa freática en distintos puntos de la Reserva. Sin embargo, de estos datos sólo se ha utilizado el registro correspondiente a diciembre de 1984 como medida comparativa entre las distintas zonas (figura 1).

Los resultados se han basado en las observaciones y capturas de individuos realizadas con una periodicidad mensual, para lo cual se han seguido dos métodos diferentes:

a) Censos nocturnos: realizados sólo para anfibios. Estos censos consistieron en el conteo de todos los individuos observados a lo largo de un recorrido de $11 \mathrm{Km}$, desde un vehículo que circulaba a una velocidad de aproximadamente $10-15 \mathrm{Km} / \mathrm{h}$. Se realizaban de noche, generalmente entre 1 ó 2 horas después de la puesta del sol, considerándose sólo los individuos que se encontraban dentro del campo de visión permitido por los faros del coche. Este método ha sido utilizado anteriormente por diversos autores (HEUSSER, 1968; STRIJBOSCH, 1980). Cada vez que se realizaba el censo se registraba la temperatura y humedad relativa del aire en el punto inicial del recorrido. 
El trayecto elegido fue el de un camino de arena que atraviesa la Reserva, comenzando en borde de marisma, que corresponde asimismo al punto de menor altitud, y finalizando en dunas estabilizadas, de manera que aumenta gradualmente la altitud conforme se recorre (figura 1). Se iniciaba tras pasar una zanja que atraviesa el camino, cubierta por unos barrotes que permiten el paso de los vehículos. Los anfibios que al atravesar el camino caían en esta zanja también eran contados y sumados a los resultados de cada censo. Asimismo el recorrido total se consideró dividido en 12 tramos de los que los 10 intermedios eran de $1 \mathrm{~km}$, y los dos extremos sumaban aproximadamente $1 \mathrm{~km}$. Cada mes se realizan dos censos en un intervalo menor de una semana, considerándose posteriormente su suma como resultados mensuales.

Los individuos observados mediante este método se identificaban y medían (longitud total, longitud pata posterior, longitud tibia y peso), si bien estos datos no se han utilizado en el presente estudio; inmediatamente después, se ponían en libertad.

b) Captura de individuos inactivos. Para ello se dispusieron, en distintos puntos de la Reserva, una serie de losas de tamaña $33 \times 33 \mathrm{~cm}$ y grosor $2,8 \mathrm{~cm}$, pretendiéndose con ello controlar a los anfibios y pequeños reptiles que las utilizaran como refugio. El sustrato del área de estudio es arenoso en su mayoría, no existiendo prácticamente piedras que puedan proporcionar cobijo.

Se dispusieron en total 17 series de 15 losetas cada una, más otra de 150 cuyas características principales y localización se describen en la tabla 1 y figura 1 .

Mensualmente se revisaba la zona cubierta por cada loseta, si bien el primer control se realizó transcurridos dos meses desde la colocación de éstas en el área de estudio. Los registros se realizaron a primeras horas de la mañana o a finales de la tarde, con el fin de capturar, tanto anfibios como reptiles.

Los individuos eran marcados mediante cortes de dedos o cicatrices en especies como Blanus cinereus o Chalcides chalcides, o bien se identificaban mediante el reconocimiento del diseño ventral, método que se utilizó para $\mathrm{Bu}$ fo calamita y Podarcis hispánica.

\section{RESULTADOS Y DISCUSIÓN}

\section{a) CENSOS}

En la totalidad de censos realizados a lo largo de los 25 meses del período de estudio, se realizaron 293 observaciones de anfibios (figura 2). La mayor actividad se detecta en los meses en que se producen las mayores precipitaciones (octubre a diciembre), existiendo una correlación significativa entre actividad y precipitación. Por el contrario, se observan pocos individuos activos durante el verano, resultando completamente negativos los censos realizados en los meses en que se produjeron las más altas temperaturas en cada uno de los años y en los que la humedad relativa no es elevada (agosto 1983 y julio 1984), lo que parece implicar la existencia de un período de estivación. Por otra parte, el período de mayor actividad, se ve interrumpido también por las bajas temperaturas, aunque en esta zona no suelen alcanzarse valores negativos (enero 1983, febrero 1984). Existe asimismo correlación significativa con las tempe- 


\section{N. ${ }^{\circ}$ ESPECIES}

LOSAS VEGETALES PREDOMINANTES

OTROS

A 15 Pinus pinea, Halimium halimifolium

B 15 Frankenia laevis Chaetopogon fasciculatus

C 15 Juncus spp, F. laevis, gramíneas

D 15 Ch. fasciculatus, Juncus spp. Borde marisma

E 15 Quercus suber, Pteridium aquilinum

F $15 \quad H$. halimifolium, Stauracanthus Matorral xerófilo genistoides

G $15 \quad P$. pinea

H 15 Q. suber, P. aquilium

I 15 H. halimifolium, Erica scorapia, Ch. fasciculatus

$\mathrm{K} 15 \quad H$. halimifolium

L 15 Rosmarinus officinalis, H. commutatum

M $15 \quad$ E. scoparia

$\mathrm{N} 15$ Juniperus phoenicea, Cladonia mediterranea, $H$. commutatum

O $15 \quad$ E. scoparia

P 15 Holoschoenus vulgaris, $H$. halimifolium, $P$. pinea

R 15 Ch. fasciculatus, Juncus spp. Juncal sobre laguna

S 15 J. phoenicea, C. mediterranea, Thymus mastichina, $H$. halimifolium

X $150 \quad P$. pinea, Ch. fasciculatus, $H$. vulgaris, $H$. halimifolium.
Interior pinar

Borde laguna marismeña

Interior juncal marisma

Helechal bajo alcornoque

Interior pinar sin sotobosque

Helechal bajo alcornoque, matorral higrofítico y xerófilo

Matorral xerófilo, higrófilo y borde laguna

Matorral xerófilo

Sobre dunas estabilizadas matorral xerófilo

Matorral higrófilo

Sabinar sobre dunas

estabilizadas

Matorral higrófilo

Zona interdunar

Sabinar sobre dunas

estabilizadas

Zona interdunar

Tabla 1. - Principales características de cada una de las series de losas colocadas para la captura de anfibios y pequeños reptiles. 
raturas máxima y mínima. Los coeficientes de correlación de Spearman para los anteriores parámetros resultaron:

$\mathrm{r}_{\mathrm{S}}($ pluviosidad $)=0,611(\mathrm{p}<0,01)$

$\mathrm{r}_{\mathrm{s}}$ (temperatura mín.) $=-0,311(\mathrm{p}<0,05)$.

$\mathrm{r}_{\mathrm{s}}$ (temperatura máx.) $=-0,577(\mathrm{p}<0,01)$.

$\mathrm{r}_{\mathrm{s}}$ (humedad rel.) $=0,334(\mathrm{p}<0,05)$.

Los censos realizados detectan la actividad terrestre de los anfibios, por lo que las especies de actividad ligada al medio acuático están escasa o nulamente representadas. Este en el caso de Rana perezi, Discoglossus galganoi (la denominación taxonómica de esta especie se encuentra actualmente en debate; en este trabajo se ha adoptado la más reciente, descrita por Capula y col, 1985) y de las dos especies de tritones: Triturus marmoratus, de la que los dos únicos datos existentes coinciden con la llegada de los individuos a las charcas, y Triturus boscai, de la que no se ha encontrado ningún ejemplar. Pleurodeles walt es también una especie cuya actividad en Doñana está muy ligada al medio acuático. Las observaciones de individuos coinciden con la formación de los medios acuáticos temporales y con su desecación poco antes del verano.

La actividad trepadora de Hyla meridionalis, así como su larga estación reproductora, que implica un largo período de asociación de los individuos machos al medio acuático (DÍAZ-PANIAGUA, 1986), hacen que esta especie también esté escasamente representada en los censos. Los individuos encontrados el primer año de estudio fueron adultos que aparecieron antes del comienzo de su período reproductor, mientras que en el segundo año se observaron individuos jóvenes recién metamorfoseados.

De los anfibios de Doñana, Pelobates cultripes, Bufo calamita y Bufo bufo son las especies de actividad típicamente terrestre. Estas sólo están asociadas al medio acuático durante la reproducción, que suele ocurrir en un período de tiempo breve, variable entre unos días y varias semanas (DÍAZPANIAGUA, 1985).

B. bufo, sin embargo, presenta muy bajas densidades en el área de estudio, por lo que sus observaciones son escasas y se limitan al inicio del período reproductor, correspondiendo a los adultos que se dirigian hacia los hábitats de reproducción.

$B$. calamita, es una especie abundante en Doñana. El mayor número de individuos observados corresponde a la época en que se producen las precipitaciones más importantes, cuando su actividad debe estar básicamente dedicada al mantenimiento, puesto que el período de reproducción no suele ocurrir hasta enero-febrero (DÍAZ-PANIAGUA, 1985). Durante el verano se aprecia un período de inactividad, no observándose ningún individuo en los censos de dicha estación en 1983.

$P$. cultripes, es el anfibio más abundante de Doñana. Su actividad se detacta prácticamente durante todo el año. Las máximas frecuencias coinciden con la época en que se producen las mayores precipitaciones, que es precisamente cuando suele iniciarse su actividad reproductora. Esto ocurre en noviembre y diciembre del primer año de estudio, y entre noviembre y enero del segundo, detectándose entonces migraciones de individuos hacia las charcas. Pasado este período, se sigue detectando actividad durante prácticamente todo el año, excepto en los casos ya comentados que coinciden con las temperaturas más extremas en el área de estudio. 


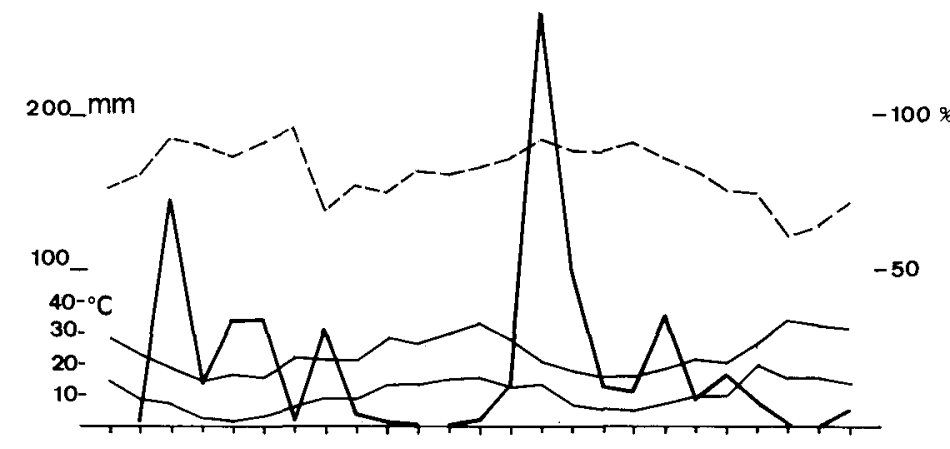

$0,5-$

R. perezi
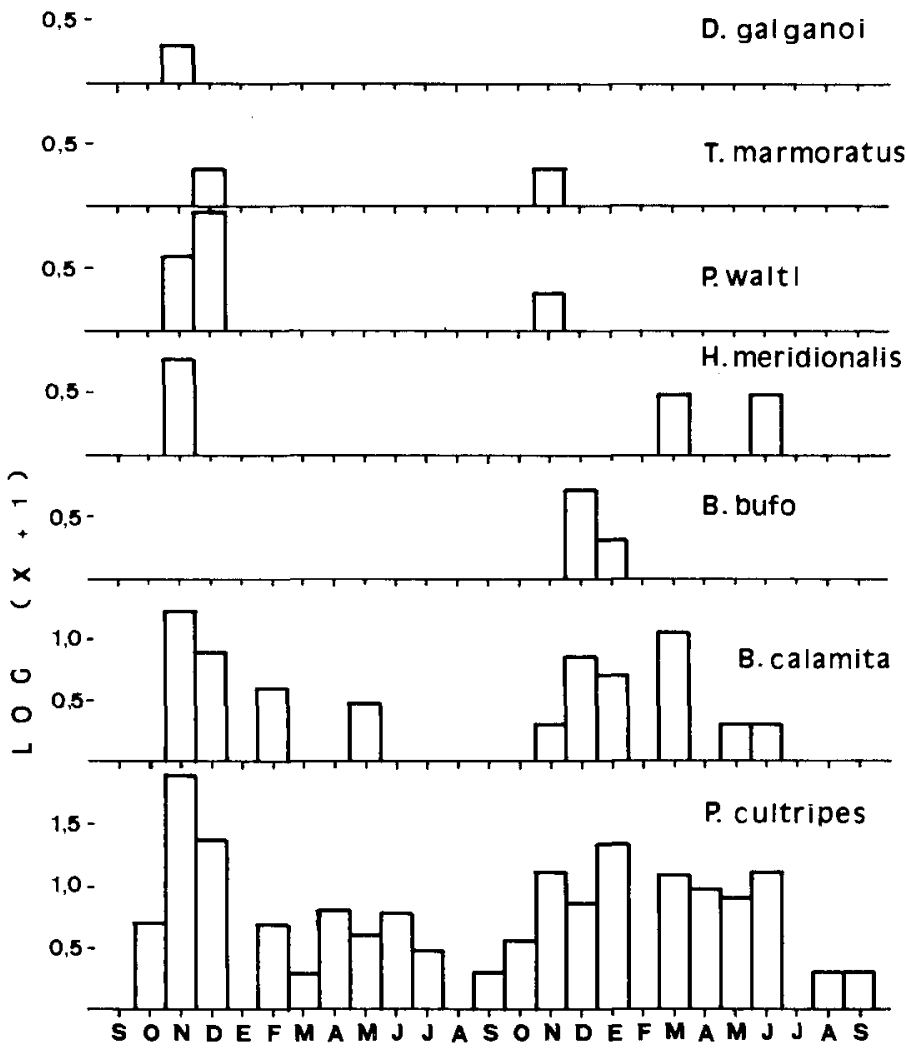

Figura 2.-Variación de la precipitación (trazo grueso), temperaturas máxima y mínima (trazo fino) y humedad relativa (trazo discontinuo) e histogramas de la frecuencia de aparición de los censos [expresado en log $(x+1)$ ] a lo largo del período de estudio. 


\begin{tabular}{|c|c|c|c|c|c|c|c|c|c|c|c|c|}
\hline & $\mathbf{0}$ & $0-1$ & $1-2$ & $2-3$ & $3-4$ & 4-5 & $5-6$ & $6-7$ & $7-8$ & 8-9 & $9-10$ & 10-11 \\
\hline$P$. cultripes & 53 & 21 & 23 & 28 & 11 & 31 & 28 & 4 & 8 & - & - & 1 \\
\hline B. calamita & 2 & 5 & 9 & 5 & 8 & 7 & 4 & 2 & 1 & 5 & 2 & 1 \\
\hline H. meridionalis & - & 1 & 1 & 3 & 1 & - & 1 & - & 1 & - & - & - \\
\hline P. waltl & 8 & 2 & 1 & 1 & 2 & 1 & - & - & - & - & - & - \\
\hline B. bufo & - & - & 1 & 2 & 1 & 1 & - & - & - & - & - & - \\
\hline R. perezi & 2 & - & - & 1 & - & - & - & - & 一 & - & - & - \\
\hline T. marmoratus & 2 & - & - & - & - & - & - & - & - & - & - & - \\
\hline D. galganoi & - & - & - & - & 1 & - & - & - & - & - & - & - \\
\hline TOTAL & 67 & 29 & 35 & 39 & 25 & 40 & 32 & 6 & 10 & 5 & 2 & 2 \\
\hline
\end{tabular}

Tabla 2.-Número de individuos de cada especie de anfibios observado según los distintos tramos del censo.

El número total de observaciones de las distintas especies a lo largo de los distintos tramos del recorrido no es uniforme (tabla 2), sino que presenta una clara disminución a partir del tramo 6-7. Es a partir de este tramo cuando se produce una mayor elevación del terreno, al atravesar el recorrido la zona de dunas estabilizadas, donde la capa freática se encuentra a mayor profundidad y apenas se forman charcas que posibiliten la reproducción de los anfibios.

En la primera zona, por el contrario se forman innumerables charcas temporales, que junto a la marisma próxima son el hábitat de reproducción por excelencia en el área. Sólo en esta zona se han encontrado, aunque con frecuencias bajas, las especies que no son típicamente terrestres, que como ya hemos comentado se detectan fundamentalmente en su migración hacia los hábitats de reproducción. Los escasos ejemplares de $B$. bufo observados también se encuentran en esta zona. En cuanto a las dos especies más abundantes, su mayor actividad terrestre denota también una mayor capacidad para alejarse de los lugares en que se concentran para la reproducción, si bien en las zonas más bajas se encuentra un mayor número de individuos.

\section{b) CAPTURAS BAJO REFUGIOS}

Los refugios artificiales que se dispusieron para la captura de individuos han proporcionado información tanto para algunas especies de anfibios como para pequeños reptiles.

La tabla 3 representa los individuos capturados a lo largo del período de estudio. El total de individuos capturados es 179, un número poco elevado en relación a la cantidad de refugios controlados.

Anfibios:

Los anfibios encontrados bajo las losas aparecen durante los dos años de estudio a partir del mes de noviembre, coincidiendo con el inicio de la actividad percibido a través de los censos. En consecuencia, los anfibios observados por este método no utilizan estos refugios para pasar un período largo de inactividad, sino que, por el contrario, éstos funcionan como un hábitat transitorio en el que se refugian ocasionalmente durante su período de actividad. 


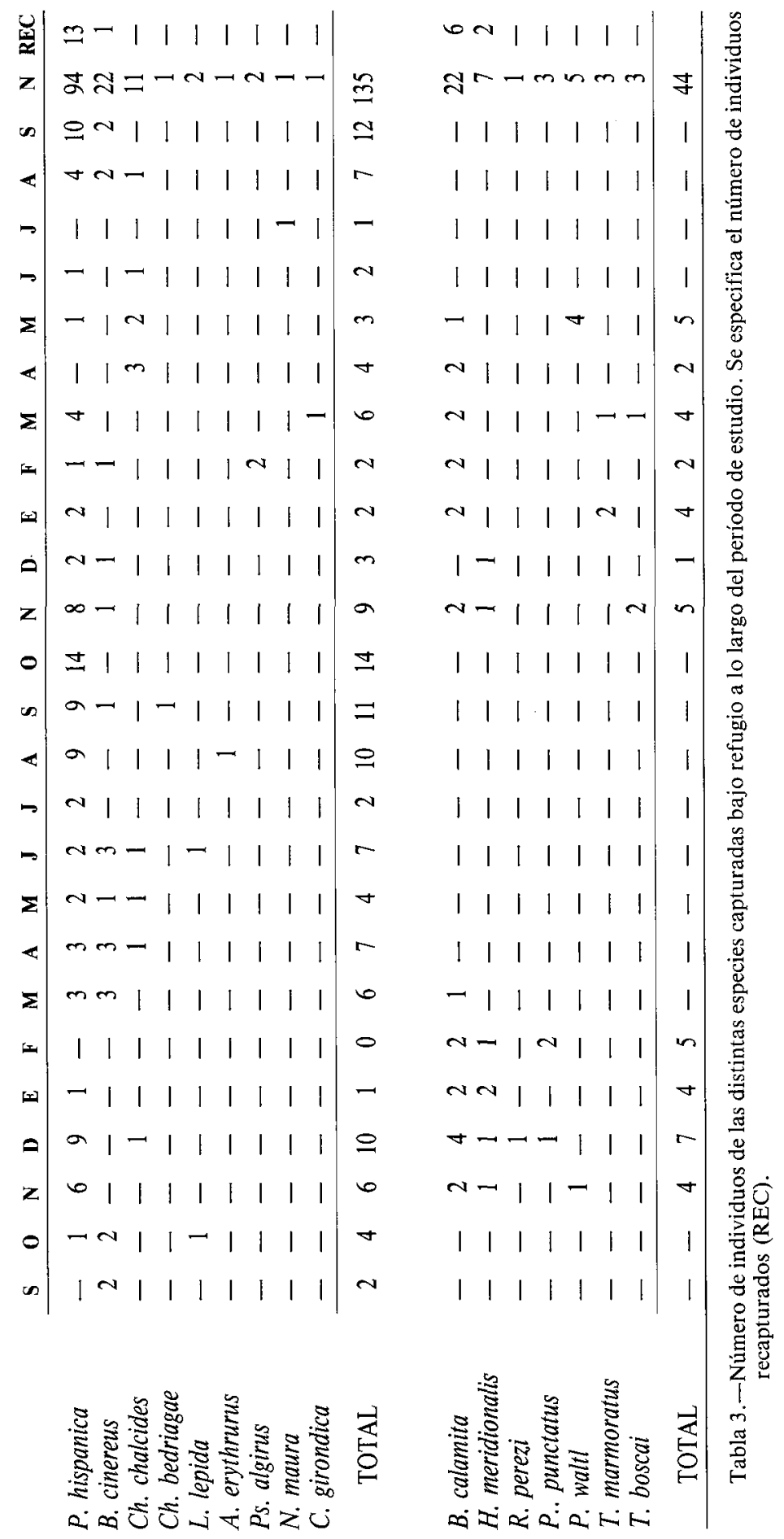


Entre las especies observadas cabe destacar el hecho de que sólo dos, $B$. calamita y $H$. meridionalis, se han registrado recapturas, que no son en todos los casos consecutivas. Esto hace pensar que puedan llegar a instalarse bajo las losas durante un período en el que su actividad se desarrolle alrededor de ellas.

De las diez especies presentes en Doñana, sólo aparecen siete. $P$. cultripes, la especie más abundante en Doñana (COLLADO y col, 1976), no llegó a utilizar las losas, lo que se atribuye a la escasa o nula necesidad de este tipo de refugios para esta especie, que en las arenas de Doñana desarrolla óptimamente su capacidad excavadora. Tampoco aparecen $B$. bufo, probablemente imposibilitado por su tamaño (aunque podrían haber sido utilizadas por los individuos jóvenes), ni $D$. galganoi. La ausencia de esta última especie es probablemente debida a su afinidad al medio acuático (KNOEPFFLER, 1962), pudiendo mantenerse en sus momentos de menor actividad en la proximidad de charcas o lagunas, igual que puede ocurrir con $R$. perezi, de la que sólo se ha encontrado un individuo. Otras especies ligadas al medio acuático son los urodelos, de los que la mayoría de los individuos observados fueron juveniles.

Las tres observaciones de $P$. puntatus corresponden a individuos adultos que se encontraban en su fase reproductiva y que se localizaron bajo losas dispuestas próximas a lugares de reproducción, en medio de una marisma seca.

Los individuos observados de $H$. meridionalis fueron adultos, que aparecieron antes del comienzo de su actividad reproductora.

$B$. calamita es la especie que mayor uso ha hecho de las losas como refugio, habiéndose llegado a capturar 16 individuos diferentes. El período de actividad detectado por este método se corresponde prácticamente con los resultados obtenidos mediante los censos.

Reptiles:

Se han registrado en total 135 observaciones de reptiles (tablas 3 y 4). La mayoría (94) corresponden a individuos de Podarcis hispánica, especie que llega a instalarse bajo las losas, usándolas como refugio habitual alrededor del cual mantiene su actividad. La mayoría de las observaciones de esta especie se sitúan en un hábitat caracterizado por la presencia de matorral xerófilo de $H$. halimifolium, lo que se relaciona con la gran extensión de este hábitat en la Reserva, sobre todo en la zona de arenas estabilizadas. Sin embargo, se aprecia también una cierta preferencia hacia las zonas limítrofes con matorral más higrófilo, donde se incrementa la riqueza de especies vegetales (tabla 3, casos $\mathrm{I}, \mathrm{H}, \mathrm{X}$ ). Se distribuye también en las zonas más altas (casos $\mathrm{N}$ y L) donde el matorral higrófilo no está presente y el xerófilo se caracteriza por la presencia de $H$. halimifolium y $C$. libanotis. Cabe también señalar que no se la encuentra en las series de la marisma (B y C) auńque sí en su borde (D).

Otra especie de la que se puede considerar que llega a instalarse bajo las losas es $\boldsymbol{B}$. cinereus, aunque el número de capturas es notablemente menor que en $P$. hispánica, y sólo se obtuvo una recaptura. Esto se atribuye a que a las tempranas horas a las que se realizaban los controles, los individuos pudieran encontrarse bajo el sustrato, en lugar de a ras del suelo bajo la losa. Sin embargo, la frecuente presencia de mudas $(\mathrm{N}=23)$ y excrementos bajo las losas corrobora su uso. Las mayores frecuencias encontradas corresponden a dos 

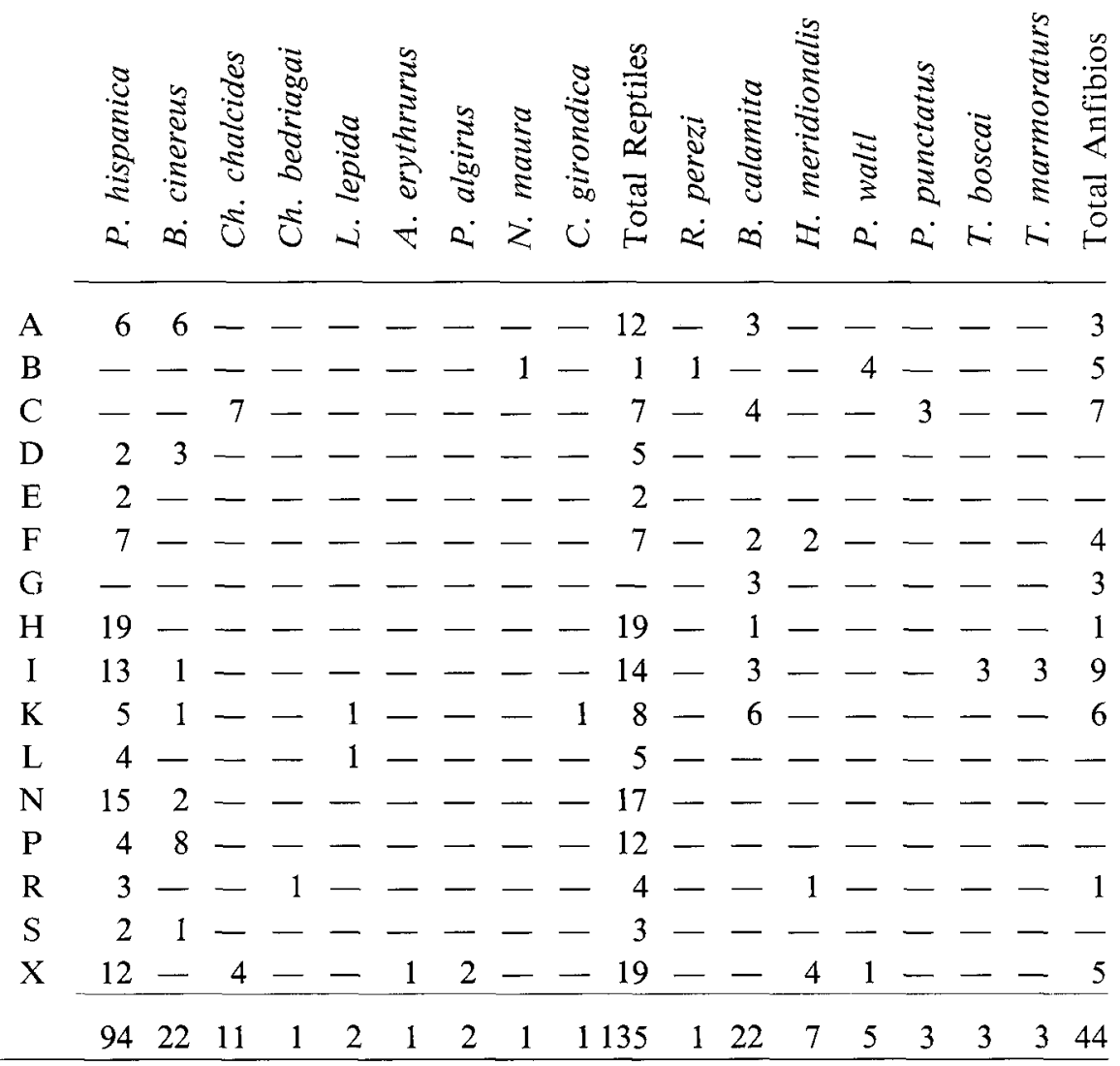

Tabla 4.-Número de individuos de cada especie observados bajo refugio en los distintos lugares controlados.

pinares (A y P) (tabla 4), en los que la presencia de matorral higrófilo y $H$. vulgaris denota que la capa freática no debe estar muy distante.

La siguiente especie en cuanto a frecuencia de aparición es $C h$. chalcides, que aparece en todas las series asociadas a pradera donde predomina Ch. fasciculatus, y a juncales de Juncus spp. o $\mathrm{H}$. vulgaris. La mayoría de los datos corresponden a una serie de losas situada en el interior de la marisma (C) en la época en que ésta se inunda, donde quedan aisladas algunas poblaciones.

De las demás especies, el número de individuos es muy pequeño. No se ha producido ninguna observación de Psammodromus hispanicus, de escasa abundancia en Doñana. Las observaciones de Psammodromus algirus y Acanthodactylus erythrurus son muy escasas, sin embargo, estas especies son muy abundantes, lo que denota que han de utilizar otro tipo de refugios, probablemente la cobertura vegetal, que les haga completamente innecesario el uso de las losas.

Por otra parte se ha dado el caso también de observaciones de especies de gran tamaño, como Lacerta lepida, Coronella girondica y Natrix maura. 
Sin embargo, de las dos primeras, las observaciones corresponden a individuos juveniles, mientras que en la tercera se trató de un individuo adulto bajo una losa sumergida en el borde de una laguna.

Los dos métodos utilizados para detectar la actividad tienen de particular que funcionan sólo con determinadas especies. El primero resulta de utilidad para detectar la actividad terrestre de los anfibios, con lo que se excluyen aquellas especies más ligadas al medio acuático; mientras que resalta las migraciones de los adultos hacia las charcas para su reproducción. El éxito del segundo método debe estas relacionado con el tipo de refugios que suelen utilizar las especies. A la hora de elegir este método, nos pareció adecuado por el hecho de que en el área de estudio no existen prácticamente piedras que puedan utilizarse como refugio. Sin embargo, sólo ha sido utilizado habitualmente por $P$. hispanica y $B$. cinereus, mientras que por parte de los anfibios, sólo por $B$. calamita y $H$. meridionalis, esta última muy escasamente. Probablemente el uso de otros refugios, como la vegetación o el subsuelo, sean una adaptación más de las otras especies, adquirida históricamente y que actualmente no se transforma por el simple hecho de disponer de otros recursos, salvo en casos excepcionales. El carácter generalista de $P$. hispánica en cuanto al uso del espacio (MELLADO, 1980), probablemente justifica la aceptación de los refugios proporcionados. Por otra parte, parece oportuno señalar que este método puede resultar muy efectivo para el control de poblaciones de aquellas especies que llegan a instalarse definitivamente en estos refugios, en este caso $B$. cinereus y $P$. hispánica. 


\section{BIBLIOGRAFÍA}

ALLIER, C., F. GONZÁLEZ BERNÁLDEZ y L. RAMÍREZ DÍAZ (1974). Mapa ecológico de la Reserva Biológica de Doñana. División Ciencias del C. S. I. C. Estación Biológica de Doñana. Sevilla.

AMAT, . A., C. MONTES DEL OLMO., L. RAMÍREZ DÍAZ, A. TORRES MARTÍNEZ (1979). Parque Nacional de Doñana. Mapa ecológico. M. ${ }^{\circ}$ Agricultura. ICONA. 24 pp.

CAPULA M., G. NASCETTI, B. LANZA, L. BULLINI Y E. G. CRESPO (1985). Morphological and genetic differentiation beween the Iberian and the other West Mediterranean Discoglossus species (Amphibia Salientia Discoglossidae). Monitore Zoologico Italiana (N. S.) 19: $69-90$.

COLLADO, E., J. CALDERÓN y M. PÉREZ (1976). Datos sobre la fauna de anfibios del Bajo Guadalquivir. Doñana Acta Vertebrata 3: 5-17.

DIAZ-PANIAGUA, C. (1985). The reproductive period of amphibians in the Biological Reserve of Doñana (SW Spain). III Ordinary General Meeting of Societas Herpetologia Europaea (en prensa).

- (1986). La reproducción de Hyla meridionalis en el suroeste de España. Doñana Acta Vertebrata 13 (en prensa).

DUELLMAN, W. E. y L. TRUEB. (1985). Biology of amphibians. Mc Grow Hill. New York. $670 \mathrm{pp}$.

GARCÍA NOVO F., J. MERINO ORTEGA, L. RAMÍREZ DÍAZ, M. RÓDENAS LARIOS, F. SANCHO ROYO, A. TORRES MARTÍNEZ, F. GONZÁLEZ BERNÁLDEZ, F. DÍAZ PINEDA, C. ALLIER, V. BRESSET y A. LACOSTE (1978). Doñana. Prospección e inventario de ecosistemas. M. ${ }^{\circ}$ Agricultura. ICONA. Monografía n. ${ }^{\circ} 18.244$ pp.

HEUSSER, H. (1968). Die Lebensweise der Erdkröte, Bufo bufo (L.); Wanderungen un Sommerquartiere. Revue suisse de Zoologie 75: 927-982.

KNOEPFFLER, L. P. (1962). Contribution a l'etude du genre Discoglossus (Amphibiens, Anoures). Vie et Milieu 13: 1-94.

MELLADO, J. (1980). Utilización del espacio en una comunidad de lacértidos del matorral mediterráneo en la Reserva Biológica de Doñana. Doñana Acta Vertebrata 7: 41-59.

SPELLERBERG, I. F. (1982). Biology of reptiles. Blackie. Glasgow \& London. 159 p.

STRIJBOSCH, H. (1980). Habitat selection by amphibians during their terrestrial phase. British Journal of Herpetology 6: 93-98.

VALVERDE, J. A. (1967). Estructura de una comunidad de vertebrados terrestres. Monografías C. S. I. C. n. ${ }^{\circ} 1218 \mathrm{pp}$.

VOGT, R. C. Y R. L. HINE (1982). Evaluation of techniques for assesment of amphibian and reptile populations in Winscosin. En: SCOTT, Jr., N. S. (ed.): Herpetological Communities: a symposium of the society for the study of amphibians and reptiles and the herpetologists' league, August 1977. Wildl. Res. Rep. 13: 201-217. 